\title{
COUNTABLE INTERPOLATION SETS AND THE GLEASON METRIC
}

\author{
D. M. OBERLIN ${ }^{1}$
}

\begin{abstract}
Countable interpolation sets of type 1 are characterized as those countable compact sets which intersect each part at most once. A metric condition is given which characterizes those countable compact sets which are interpolation sets for logmodular function algebras.
\end{abstract}

We present here two results which deal with the relationships between the Gleason "metric" and the sets of interpolation for certain function algebras. The first of these characterizes those countable compact subsets of maximal ideal spaces which are interpolation sets of type 1, while the second characterizes those countable compact sets which are sets of interpolation for logmodular function algebras. The main tools of this investigation are the decomposition theorem for measures orthogonal to a function algebra, the embedding theorem of Wermer, the characterization of interpolating sequences for $H^{\infty}$ of the unit disc, and a corollary to a theorem of Hoffman and Wermer. Our result for logmodular algebras extends a well-known characterization of the countable interpolation sets for the disc algebra.

We first establish some terminology. We shall constantly cite [1], the book of Stout, as a reference for known facts about function algebras, and we refer the reader to this source for definitions of terms with which he may be unfamiliar. Let $A$ be a function algebra with maximal ideal space $\mathscr{M}_{A}$. Let $x$ and $y$ belong to $\mathscr{M}_{A}$. Then $\psi(x, y)$, the Gleason "metric" evaluated at the ordered pair $(x, y)$, is defined to be the supremum of the numbers $|F(y)|$ where $F$ varies over all functions in the unit ball of $A$ which vanish at $x . \psi(x, y)=1$ if and only if $x$ and $y$ belong to different Gleason parts. Now let $K \subseteq \mathscr{M}_{A}$ be compact. $K$ is an interpolation set for $A$ if every continuous function on $K$ is the restriction to $K$ of some function in $A$.

Received by the editors December 18, 1972 and, in revised form, April 30, 1973. AMS (MOS) subject classifications (1970). Primary 46J10.

Key words and phrases. Function algebra, interpolation set, part, Gleason "metric".

1 This research was supported by an NDEA Title IV graduate fellowship.

(c) American Mathematical Society 1974 
When this is so there exists some constant $M<\infty$ such that, given $F \in$ $C(K)$ and $\varepsilon>0$, there exists $g \in A$ which extends $F$ and has $\|g\| \rightrightarrows$ $(M+\varepsilon)\|F\|$. We describe this situation by saying that $K$ is an interpolation set of type $M$.

Now let $K \subseteq \mathscr{M}_{A}$ be an interpolation set of type 1 and let $x$ and $y$ be distinct elements of $K$. Then there exists, for $\varepsilon>0, F \in A$ with $F(x)=0$, $F(y)=1-\varepsilon,\|F\| \leqq 1$. Hence $\psi(x, y)=1$ and so $K$ intersects each Gleason part at most once. Our first theorem states that a converse to this observation holds for countable $K$.

THEOREM 1. Let $K \subseteq \mathscr{M}_{A}$ be a countable compact set which intersects each Gleason part at most once. Then $K$ is an interpolation set of type 1.

Proof. Let $\lambda$ be a measure on $\mathscr{M}_{A}$ which is orthogonal to $A$. By a result of Glicksberg (p. 208 in [1]) it suffices to show

$$
\|\lambda\|_{K} \leqq\|\lambda\|_{\mathscr{M}_{\Delta} \backslash K}
$$

Let $K=\left\{x_{i}\right\}_{i=1}^{\infty}$. Then to show (1) it suffices to show that

$$
\begin{aligned}
\sum_{i=1}^{n_{0}}\left|\lambda\left(\left\{x_{i}\right\}\right)\right| & =\|\lambda\|_{\left\{x_{1}, \cdots, x_{n_{0}}\right\}} \leqq\|\lambda\|_{\mathscr{M}_{\Delta} \mid\left\{x_{1} \cdots, x_{n_{0}}\right\}} \\
& =\|\lambda\|_{\mathscr{M}_{\Delta} \mid K}+\sum_{i=n_{0}+1}^{\infty}\left|\lambda\left(\left\{x_{i}\right\}\right)\right|
\end{aligned}
$$

holds for every $n_{0}$. Let $G=\left\{x_{1}, \cdots, x_{n_{0}}\right\}$.

By the decomposition theorem for orthogonal measures (p. 258 in [1]), there exists a sequence $\varphi_{n}, n=1, \cdots$, of elements in $\mathscr{M}_{A}$ which lie in distinct Gleason parts such that for some representing measure $\mu_{n}$ for $\varphi_{n}$, and some Borel $E \subseteq \mathscr{M}_{A}, \lambda=\sum_{n=1}^{\infty} \lambda_{n}+\left.\lambda\right|_{E}$, where $\lambda_{n}$ is absolutely continuous with respect to $\mu_{n}$ and $\left.\lambda\right|_{E}$ is singular with respect to every representing measure for every point in $\mathscr{M}_{A}$. Furthermore each $\lambda_{n}$ and $\left.\lambda\right|_{E}$ is orthogonal to $A$. Now since the $\mu_{n}$ are all pairwise mutually singular (p. 165 of [1]) and are all singular with respect to $\left.\lambda\right|_{E}$, we have $\|\lambda\|_{S}=\sum_{n=1}^{\infty}\left\|\lambda_{n}\right\|_{S}+\|\lambda\|_{E \cap S}$ for any Borel $S \subseteq \mathscr{M}_{A}$, so to show (2) it actually suffices to show

$$
\sum_{i=1}^{\infty}\left\|\lambda_{i}\right\|_{G}+\|\lambda\|_{E \cap G} \leqq \sum_{i=1}^{\infty}\left\|\lambda_{i}\right\|_{\mathscr{M}_{\Delta} \backslash G}+\|\lambda\|_{E \backslash G}
$$

Since $\left.\lambda\right|_{E}$ is singular with respect to every point mass on $\mathscr{M}_{A},\|\lambda\|_{E \cap G}=0$, so we have

$$
\|\lambda\|_{E \cap G} \leqq\|\lambda\|_{E \backslash G} .
$$


Now, after reordering the $\lambda_{i}$ and $\varphi_{i}$, there is a nonnegative integer $k \leqq n_{0}$ such that for $j \leqq k, x_{j}$ and $\varphi_{j}$ are in the same part, while for $j>k, \varphi_{j}$ is not in a part with any of the $x_{i}\left(1 \leqq i \leqq n_{0}\right)$. Fix $j>k$. Since $\varphi_{j}$ is not in the same part as any $x_{i}\left(1 \leqq i \leqq n_{0}\right), \mu_{j}$ is singular with respect to each of the point masses $\delta_{x_{i}}\left(1 \leqq i \leqq n_{0}\right)$.

Since $\lambda_{j}$ is absolutely continuous with respect to $\mu_{j},\left\|\lambda_{j}\right\|_{G}=0$, so we have

$$
\left\|\lambda_{j}\right\|_{G} \leqq\left\|\lambda_{j}\right\|_{\mathscr{M}_{A} \mid G}
$$

for $j>k$. If, on the other hand, $j \leqq k$, then $x_{i}$ and $\varphi_{j}$ belong to different parts for $1 \leqq i \leqq n_{0}$ and $i \neq j$, so, as above, $\left\|\lambda_{j}\right\|_{G}=\left|\lambda_{j}\left(\left\{x_{j}\right\}\right)\right|$. If $\left|\lambda_{j}\left(\left\{x_{j}\right\}\right)\right|=0$ then (5) is clear. If $\left|\lambda_{j}\left(\left\{x_{j}\right\}\right)\right| \neq 0$, then, since $\lambda_{j}$ is orthogonal to $A$, $\lambda_{j}\left(\left\{x_{j}\right\}\right) F\left(x_{j}\right)=-\int_{\mathscr{M}_{A} \mid\left\{x_{j}\right\}} F d \lambda_{j}$ for every $F$ in $A$. But $\left.\lambda_{j}\right|_{\mathscr{M}_{A} \mid\left\{x_{j}\right\}}=\left.\lambda_{j}\right|_{\mathscr{M}_{A} \mid G}$, since $\lambda_{j}\left(\left\{x_{i}\right\}\right)=0$ if $i \neq j$, so $-\left.\left(1 / \lambda_{j}\left(\left\{x_{j}\right\}\right)\right) \lambda_{j}\right|_{G}$ is a complex representing measure for $x_{j}$. Hence

$$
\left(1 /\left|\lambda_{j}\left(\left\{x_{j}\right\}\right)\right|\right)\left\|\lambda_{j}\right\|_{\mathscr{M}_{\Lambda} \mid G} \geqq 1,
$$

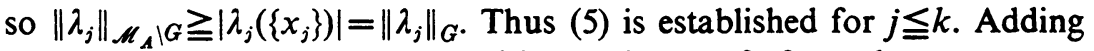
(4) and (5) for all $j$ we get (3) and hence the proof of our theorem.

Our second theorem deals with countable interpolation sets for logmodular function algebras.

THEOREM 2. Let $A$ be a logmodular function algebra and let $K=$ $\left\{x_{1}, \cdots\right\}$ be a countable compact subset of $\mathscr{M}_{A}$.

(a) If there exists $\delta>0$ such that for every $x_{j_{0}} \in K$ we have

$$
\prod_{j=1 ; j \neq j_{0}}^{\infty} \psi\left(x_{j_{0}}, x_{j}\right) \geqq \delta,
$$

then $K$ is an interpolation set of type $\leqq 2(1-2 \log \delta) / \delta^{5}$ for $A$.

(b) If $K$ is an interpolation set of type $M$ for $A$, then

for every $x_{j_{0}} \in K$.

$$
\prod_{j=1 ; j \neq j_{0}}^{\infty} \psi\left(x_{j_{0}}, x_{j}\right) \geqq 1 / M
$$

Proof of (a). Let $\lambda$ be a measure on $\mathscr{M}_{A}$ which is orthogonal to $A$. Then, as before, it suffices to show

$$
\|\lambda\|_{K} \leqq M\|\lambda\|_{\mathscr{M}_{\Lambda} \backslash K}
$$

where $M=2(1-2 \log \delta) / \delta^{5}$. Let $\lambda=\sum_{i} \lambda_{i}+\left.\lambda\right|_{E}$ be a decomposition of $\lambda$ as in Theorem 1 so that $\lambda_{i}$ is absolutely continuous with respect to some representing measure $\mu_{i}$ for the element $\varphi_{i} \in \mathscr{M}_{A}$, and, if $P_{i}$ is the part to 
which $\varphi_{i}$ belongs, $P_{i}=P_{j}$ only if $i=j$. Then

$$
\begin{aligned}
\|\lambda\|_{K} & =\sum_{i}\left\|\lambda_{i}\right\|_{K}+\|\lambda\|_{E \cap K}=\sum_{i}\left(\sum_{j}\left|\lambda_{i}\left(\left\{x_{j}\right\}\right)\right|\right)+\sum_{j}|\lambda|_{E}\left(\left\{x_{j}\right\}\right) \mid \\
& =\sum_{i} \sum_{j}\left|\lambda_{i}\left(\left\{x_{j}\right\}\right)\right|=\sum_{i} \sum_{x \in S_{i}}\left|\lambda_{i}(\{x\})\right|, \quad \text { where } S_{i}=P_{i} \cap K,
\end{aligned}
$$

since $\left.\lambda\right|_{E}$ is singular with respect to every point mass and since $\lambda_{i}(\{x\})=0$ if $x \notin P_{i}$.

Since $\|\lambda\|_{\mathscr{M}_{A} \backslash K} \geqq \sum_{i}\left\|\lambda_{i}\right\|_{\mathscr{M}_{\Delta} \backslash K}$, to show (1) it suffices by (2) to prove that for every $i$,

$$
\sum_{x \in S_{i}}\left|\lambda_{i}(\{x\})\right| \leqq M\left\|\lambda_{i}\right\|_{\mathscr{M}_{A} \backslash K}
$$

We distinguish two cases. If $S_{i}$ consists of just one point $x$, then either $\lambda_{i}(\{x\})=0$ and (3) is clear, or $\lambda_{i}(\{x\}) \neq 0$ and so (as seen in the proof of Theorem 1) $-\left.\lambda_{i}(\{x\})^{-1} \lambda_{i}\right|_{\mathscr{M}_{\Lambda} \backslash K}$ is a complex representing measure for $x$ and therefore has norm $\geqq 1$. Since $M \geqq 1$, (3) follows if $S_{i}$ consists of just one point.

If $S_{i}$ consists of more than one point, then the part $P_{i}$ is nontrivial. We shall use Wermer's embedding theorem (p. 170 of [1]) to prove that each finite subset $E_{i}$ of $S_{i}$ is an interpolation set of type $\leqq M$ for $A$, whence

$$
\sum_{x \in E_{i}}\left|\lambda_{i}(\{x\})\right| \leqq M\left\|\lambda_{i}\right\|_{\mathscr{M}_{A} \mid E_{i}}
$$

by Glicksberg's theorem. Since $E_{i}$ is arbitrary, (3) follows from (4).

There is a one-to-one map $\tau$ of the open unit disc $U$ onto $P_{i}$ such that $f \circ \tau$ is an analytic on $U$ if $f \in A$. Fix a finite set $E_{i}=\left\{y_{1}, \cdots, y_{k}\right\} \subseteq S_{i}$. Let $\omega_{1}, \cdots, \omega_{k}$ in $U$ be such that $\tau\left(\omega_{i}\right)=y_{i}$. Schwarz's lemma from complex function theory tells us that if $F \in H^{\infty}(U), z, \omega \in U$, and $F(\omega)=0$, then $|F(z)| \leqq\|F\| \circ|B(z, \omega)|$ where $B(z, \omega)=(z-\omega) /(1-\bar{\omega} z)$. Hence if $f \in A$ and $f\left(y_{i_{1}}\right)=0$, then $\left|f\left(y_{i_{2}}\right)\right|=\left|f \circ \tau\left(\omega_{i_{2}}\right)\right| \leqq\|f\| \circ\left|B\left(\omega_{i_{2}}, \omega_{i_{1}}\right)\right|$ since $f \circ \tau \in$ $H^{\infty}(U)$. Thus $\psi\left(y_{i_{1}}, y_{i_{2}}\right) \leqq\left|B\left(\omega_{i_{2}}, \omega_{i_{1}}\right)\right|$. Hence, for any $i_{0} \in\{1, \cdots, k\}$,

$$
\delta \leqq \prod_{j=1 ; x_{j} \neq y_{i_{0}}}^{\infty} \psi\left(y_{i_{0}}, x_{j}\right) \leqq \prod_{i=1 ; i \neq i_{0}}^{k} \psi\left(y_{i_{0}}, y_{i}\right) \leqq \prod_{i=1 ; i \neq i_{0}}^{k}\left|B\left(\omega_{i_{0}}, \omega_{i}\right)\right|
$$

This implies, by p. 203 of [2], that $\left\{\omega_{1}, \cdots, \omega_{k}\right\}$ is an interpolation set of type $\leqq M$ for $H^{\infty}(U)$, and the proof of (a) will be finished when we show that $E_{i}$ is an interpolation set of type $\leqq M$ for $A$. So let $h \in C\left(E_{i}\right)$ and fix $\varepsilon>0$. Then $h \circ \tau \in C\left(\left\{\omega_{1}, \cdots, \omega_{k}\right\}\right)$, so there exists an $\tilde{h} \in H^{\infty}(U)$ with $\|\tilde{h}\| \leqq(M+\varepsilon)\|h\|$ and such that $\tilde{h}$ interpolates to $h \circ \tau$ on $\left\{\omega_{1}, \cdots, \omega_{k}\right\}$. Now $\tilde{h} \circ \tau^{-1}$ is a bounded analytic function on $P_{i}$, so by Theorem 29 on 
p. 154 of [3] which is a corollary to a theorem of Hoffman and Wermer, there exists a sequence $\left\{g_{n}\right\}$ in $A$ with $\left\|g_{n}\right\| \leqq\|\tilde{h}\|$ and $g_{n} \rightarrow \tilde{h} \circ \tau^{-1}$ pointwise on $P_{i}$.

Now $E_{i}$ is a finite set and so is an interpolation set of some type $C<\infty$. Pick $g_{n_{0}} \in A$ with $\left|g_{n_{0}}\left(y_{i}\right)-\tilde{h} \circ \tau^{-1}\left(y_{i}\right)\right|<\varepsilon$ for $i=1, \cdots, k$. Then there exists a function $g \in A$ with $g\left(y_{i}\right)=\tilde{h} \circ \tau^{-1}\left(y_{i}\right)-g_{n_{0}}\left(y_{i}\right)$ for $i=1, \cdots, k$ and $\|g\| \leqq(C+\varepsilon) \varepsilon$. Then $g_{n_{0}}+g$ is a function in $A$ which interpolates to $h$ on $E_{i}$ and has

$\left\|g_{n_{0}}+g\right\| \leqq\left\|g_{n_{0}}\right\|+\|g\| \leqq\|\tilde{h}\|+(C+\varepsilon) \varepsilon \leqq(M+\varepsilon)\|h\|+(C+\varepsilon) \varepsilon$.

Since $\varepsilon$ was arbitrary, $E_{i}$ is an interpolation set of type $M$ and we are done with the proof of (a).

Proof OF (b). To show (b) it suffices to show that for $x_{j_{1}}, \cdots, x_{j_{n}}$ in the same part as $x_{j_{0}}, \prod_{i=1}^{n} \psi\left(x_{j_{0}}, x_{j_{i}}\right) \geqq 1 / M$. Let $P$ be the part to which $x_{j_{0}}, \cdots, x_{j_{n}}$ belong and let $\tau: U \rightarrow P$ be an embedding map as before. Let $\omega_{0}, \cdots, \omega_{n}$ be the elements of $U$ such that $\tau\left(\omega_{i}\right)=x_{j_{i}}$. Since $\left\{x_{j_{0}}, \cdots\right.$, $\left.x_{j_{n}}\right\}$ is an interpolation set of type $M$ for $A,\left\{\omega_{0}, \cdots, \omega_{n}\right\}$ is an interpolation set of type $M$ for $H^{\infty}(U)$ because $f \circ \tau \in H^{\infty}$ if $f \in A$. Thus, by the remarks on p. 196 of [2],

$$
\prod_{i=1}^{n}\left|\frac{\omega_{0}-\omega_{i}}{1-\bar{\omega}_{i} \omega_{0}}\right| \geqq \frac{1}{M}
$$

and it is only necessary to show that $\psi\left(x_{j_{0}}, x_{j_{i}}\right) \geqq\left|\left(\omega_{i}-\omega_{0}\right) /\left(1-\bar{\omega}_{0} \omega_{i}\right)\right|$. But the function $F$ defined by $F(z)=\left(z-\omega_{0}\right) /\left(1-\bar{\omega}_{0} z\right)$ is in the unit ball of $H^{\infty}(U)$, so, by Theorem 29 on p. 154 of [3] again, there exists a sequence $\left\{g_{n}\right\}$ in the unit ball of $A$ with

$$
g_{n}\left(x_{j_{0}}\right) \rightarrow F \circ \tau^{-1}\left(x_{j_{0}}\right)=0, \quad g_{n}\left(x_{j_{i}}\right) \rightarrow F \circ \tau^{-1}\left(x_{j_{i}}\right)=\frac{\omega_{i}-\omega_{0}}{1-\bar{\omega}_{0} \omega_{i}} .
$$

Hence it is clear that $\psi\left(x_{j_{0}}, x_{j_{i}}\right) \geqq\left|\left(\omega_{i}-\omega_{0}\right) /\left(1-\bar{\omega}_{0} \omega_{i}\right)\right|$ as desired.

REMARKS. (1) The example of the unit circle, which meets each Gleason part for the disk algebra at most once but is not a set of interpolation, shows that some hypothesis such as countability is needed in Thoerem 1.

(2) For certain logmodular algebras, there are no countable compact sets of interpolation in the maximal ideal space. Thus, it has been proved by A. Dufresnoy, Sur les compacts d'interpolation du spectre de $H^{\infty}(D)$, Studia Math. 43 (1972), 235-244, that the finite sets are the only compact metrizable sets of interpolation in the spectrum of $H^{\infty}$, and that the same is true for any uniform algebra, with Silov boundary an $F$-space, having the unique representing measures property. 


\section{BIBLIOGRAPHY}

1. E. Stout, Theory of uniform algebras, Bogden and Quigley, Belmont, 1971.

2. K. Hoffman, Banach spaces of analytic functions, Prentice-Hall Series in Modern Analysis, Prentice-Hall, Englewood Cliffs, N.J., 1962. MR 24 \#A2844.

3. G. Leibowitz, Lectures on complex function algebras, Scott, Foresman and Co., Glenview, Ill., 1970.

Department of Mathematics, University of Maryland, College Park, MARYLAND 20742 